\title{
QUATERNION ALGEBRAS and INVARIANTS of VIRTUAL KNOTS and LINKS \\ II: The Hyperbolic Case
}

\author{
STEPHEN BUDDEN, ROGER FENN ${ }^{1}$ \\ ${ }^{1}$ School of Mathematical Sciences, University of Sussex \\ Falmer, Brighton, BN1 9RH, England \\ e-mail addresses: rogerf@sussex.ac.uk, stevie_hair@yahoo.com
}

ABSTRACT

Let $A, B$ be invertible, non-commuting elements of a ring $R$. Suppose that $A-1$ is also invertible and that the equation

$$
[B,(A-1)(A, B)]=0
$$

called the fundamental equation is satisfied. Then an invariant $R$-module is defined for any diagram of a (virtual) knot or link. Solutions in the classic quaternion case have been found by Bartholomew, Budden and Fenn. Solutions in the generalised quaternion case have been found by Fenn in an earlier paper. These latter solutions are only partial in the case of $2 \times 2$ matrices and the aim of this paper is to provide solutions to the missing cases.

\section{Introduction}

Let $A, B$ be invertible, non-commuting elements of a ring $R$. Suppose that $A-1$ is also invertible. Our aim is to find solutions to the equation

$$
A^{-1} B^{-1} A B-B^{-1} A B=B A^{-1} B^{-1} A-A,
$$

called the fundamental equation. In this case an invariant $R$-module is defined for any diagram of a (virtual) knot or link. Solutions in the classic quaternion case have been found, see $[\mathbf{B F}],[\mathbf{B u F}]$. Solutions in the generalised quaternion case have been found, see $[\mathbf{F}]$. These are only partial in the case of $2 \times 2$ matrices; that is sufficient but not necessary conditions were given. The matrices satisfying these conditions were called matching. The aim of this paper is to provide solutions of the missing matrices: the mismatching or hyperbolic matrices. This means that this paper together with earlier papers provides all $2 \times 2$ matrix solutions to the fundamental equation. These solutions may be summed up with the help of the following theorem.

Theorem 1.1 Suppose $A, B$ are two non-commuting $2 \times 2$ matrix solutions of the fundamental equation. Then either

$$
\operatorname{tr}(A)=\operatorname{det}(A) \text { and } \operatorname{tr}\left(A B^{-1}\right)=0
$$

or the pair $A, B$ are similar to a pair of the form

$$
A=\left(\begin{array}{cc}
a_{0}+a_{3} & 2 a_{1} \\
0 & a_{0}-a_{3}
\end{array}\right) \quad B=\left(\begin{array}{cc}
\frac{2 b_{3}}{a_{0}-a_{3}} & 2 b_{1} \\
0 & 2 b_{3}\left(\frac{1}{a_{0}-a_{3}}-2\right)
\end{array}\right)
$$


There are a number of sporadic $n \times n$ matrix solutions to the fundamental equation which will appear in a further paper with V. Turaev. However these are almost certainly not complete and finding the general $n \times n$ solution is probably very hard.

With these solutions whole new families of invariant modules and polynomials of virtual knots and links are defined. An appendix where these are calculated from the examples in the table of N. Kamada will be put on the web.

Many of the conventions and notation can be found in $[\mathbf{F}]$. We will reproduce the details necessary for the understanding of this paper and leave fine details for the interested reader in $[\mathbf{F}]$.

\section{Generalised Quaternions}

Let $F$ be a field of characteristic not equal to 2. Pick two non-zero elements $\lambda, \mu$ in $F$. Let $\left(\frac{\lambda, \mu}{F}\right)$ denote the algebra of dimension 4 over $F$ with basis $\{1, \mathbf{i}, \mathbf{j}, \mathbf{k}\}$ and relations $\mathbf{i}^{2}=\lambda, \mathbf{j}^{2}=\mu, \mathbf{i} \mathbf{j}=-\mathbf{j} \mathbf{i}=\mathbf{k}$. The multiplication table is given by

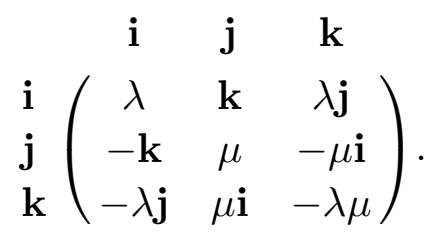

We will only consider the case $\lambda=-1$ and $\mu=1$. In this case the algebra is the ring of $2 \times 2$ matrices, $M_{2}(F)=\left(\frac{-1,1}{F}\right)$ or $\left(\frac{1,1}{F}\right)$. This is the only quaternion algebra with zero divisors or non-zero isotropic elements.

The generators of $\left(\frac{-1,1}{F}\right)$ are, together with the identity, the Pauli matrices

$$
\mathbf{i}=\left(\begin{array}{cc}
0 & 1 \\
-1 & 0
\end{array}\right), \mathbf{j}=\left(\begin{array}{ll}
0 & 1 \\
1 & 0
\end{array}\right), \mathbf{k}=\left(\begin{array}{cc}
1 & 0 \\
0 & -1
\end{array}\right)
$$

By an abuse of notation we will often confuse the scalar matrix $\left(\begin{array}{ll}\nu & 0 \\ 0 & \nu\end{array}\right)$ with the corresponding field element $\nu$.

We denote general $2 \times 2$ matrices by capital roman letters such as $A, B, \ldots$. If the trace of a matrix is zero then we denote it by bold face lower case, $\mathbf{a}, \mathbf{b}, \ldots$ etc. Field elements, (scalars) will be denoted by lower case roman letters such as $a, b, \ldots$ and lower case greek letters such as $\alpha, \beta, \ldots$. Therefore any $2 \times 2$ matrix can be written uniquely as

$$
A=a_{0}+\mathbf{a} .
$$


We call a the traceless part of $A$. Any traceless matrix can be written as a unique three dimensional linear combination of the Pauli matrices.

The conjugate of $A$ (sometimes called the adjugate) is $\bar{A}=a_{0}-\mathbf{a}$. In symbols

$$
\overline{\left(\begin{array}{ll}
a & b \\
c & d
\end{array}\right)}=\left(\begin{array}{cc}
d & -b \\
-c & a
\end{array}\right)
$$

The determinant of $A$ is $\operatorname{det}(A)=A \bar{A}$ and the trace of $A$ is $\operatorname{tr}(A)=A+\bar{A}$.

Conjugation is an anti-isomorphism of order 2. That is it satisfies

$$
\overline{A+B}=\bar{A}+\bar{B}, \quad \overline{A B}=\bar{B} \bar{A}, \quad \overline{a A}=a \bar{A}, \quad \overline{\bar{A}}=A
$$

Also $\bar{A}=A$ if and only if $A$ is a scalar and $\bar{A}=-A$ if and only if $A$ has trace zero.

The determinant is a scalar satisfying $\operatorname{det}(A B)=\operatorname{det}(A) \operatorname{det}(B)$. We will denote the set of values of the determinant function by $\mathcal{N}$. It is a multiplicatively closed subset of $F$ and $\mathcal{N}^{*}=\mathcal{N}-\{0\}$ is a multiplicative subgroup of $F^{*}$. An element $A$ has an inverse if and only if $\operatorname{det}(A) \neq 0$ in which case $A^{-1}=\operatorname{det}(A)^{-1} \bar{A}$.

The trace of a $2 \times 2$ matrix is twice its scalar part. A general matrix can be written uniquely as

$$
\left(\begin{array}{ll}
\alpha & \beta \\
\gamma & \delta
\end{array}\right)=\frac{1}{2}[(\alpha+\delta)+(\beta-\gamma) \mathbf{i}+(\beta+\gamma) \mathbf{j}+(\alpha-\delta) \mathbf{k}]
$$

Conversely

$$
A=a_{0}+a_{1} \mathbf{i}+a_{2} \mathbf{j}+a_{3} \mathbf{k}=\left(\begin{array}{cc}
a_{0}+a_{3} & a_{2}+a_{1} \\
a_{2}-a_{1} & a_{0}-a_{3}
\end{array}\right)
$$

Conjugation is

$$
\bar{A}=\operatorname{adj} A=\left(\begin{array}{cc}
\delta & -\beta \\
-\gamma & \alpha
\end{array}\right)=\left(\begin{array}{cc}
a_{0}-a_{3} & -a_{2}-a_{1} \\
a_{1}-a_{2} & a_{0}+a_{3}
\end{array}\right)
$$

and the determinant is

$$
\operatorname{det} A=\alpha \delta-\beta \gamma=a_{0}^{2}+a_{1}^{2}-a_{2}^{2}-a_{3}^{2}
$$

The scalar part of $A$ is $a_{0}=\operatorname{tr} A / 2=(\alpha+\delta) / 2$ and the traceless part is

$$
\left(\begin{array}{cc}
a_{3} & a_{2}+a_{1} \\
a_{2}-a_{1} & -a_{3}
\end{array}\right)=\left(\begin{array}{cc}
(\alpha-\delta) / 2 & \beta \\
\gamma & (\delta-\alpha) / 2
\end{array}\right)
$$




\subsection{Various Multiplications on $2 \times 2$ Matrices}

Let $A, B$ be two $2 \times 2$ matrices. There is a bilinear form given by

$$
A \cdot B=\frac{1}{2}(A \bar{B}+B \bar{A})=\frac{1}{2}(\bar{A} B+\bar{B} A)=\frac{1}{2} \operatorname{tr}(A \bar{B}) .
$$

The corresponding quadratic form is $\operatorname{det}(A)$. Let $\mathbf{a}, \mathbf{b}$ be traceless $2 \times 2$ matrices. Then

$$
\mathbf{a b}=-\mathbf{a} \cdot \mathbf{b}+\mathbf{a} \times \mathbf{b}
$$

where $\mathbf{a} \cdot \mathbf{b}$ is the restriction of the bilinear form and $\mathbf{a} \times \mathbf{b}$ is the cross product. The cross product has the usual rules of bilinearity and skew symmetry. The triple cross product expansion

$$
\mathbf{a} \times(\mathbf{b} \times \mathbf{c})=(\mathbf{c} \cdot \mathbf{a}) \mathbf{b}-(\mathbf{b} \cdot \mathbf{a}) \mathbf{c}
$$

is easily verified. The scalar triple product is

$$
[\mathbf{a}, \mathbf{b}, \mathbf{c}]=\mathbf{a} \cdot(\mathbf{b} \times \mathbf{c})=-\left|\begin{array}{lll}
a_{1} & a_{2} & a_{3} \\
b_{1} & b_{2} & b_{3} \\
c_{1} & c_{2} & c_{3}
\end{array}\right|
$$

from which all the usual rules can be deduced. Here $\mathbf{a}=a_{1} \mathbf{i}+a_{2} \mathbf{j}+a_{3} \mathbf{k}$ etc.

If

$$
A=\left(\begin{array}{ll}
\alpha_{1} & \alpha_{2} \\
\alpha_{3} & \alpha_{4}
\end{array}\right) \text { and } B=\left(\begin{array}{ll}
\beta_{1} & \beta_{2} \\
\beta_{3} & \beta_{4}
\end{array}\right) \text { then } A \cdot B=\frac{1}{2}\left(\alpha_{1} \beta_{4}-\alpha_{2} \beta_{3}-\alpha_{3} \beta_{2}+\alpha_{4} \beta_{1}\right)
$$

If $\mathbf{a}=\left(\begin{array}{cc}\alpha_{1} & \alpha_{2} \\ \alpha_{3} & -\alpha_{1}\end{array}\right)$ and $\mathbf{b}=\left(\begin{array}{cc}\beta_{1} & \beta_{2} \\ \beta_{3} & -\beta_{1}\end{array}\right)$ are traceless then

$$
\mathbf{a} \cdot \mathbf{b}=-\alpha_{1} \beta_{1}-\left(\alpha_{2} \beta_{3}+\alpha_{3} \beta_{2}\right) / 2
$$

and

$$
\mathbf{a} \times \mathbf{b}=\left(\begin{array}{cc}
\left(\alpha_{2} \beta_{3}-\alpha_{3} \beta_{2}\right) / 2 & \alpha_{1} \beta_{2}-\alpha_{2} \beta_{1} \\
\alpha_{3} \beta_{1}-\alpha_{1} \beta_{3} & \left(\alpha_{3} \beta_{2}-\alpha_{2} \beta_{3}\right) / 2
\end{array}\right)
$$

\subsection{Dependancy Criteria}

In this subsection we will consider conditions for sets of $2 \times 2$ matrices to be linearly dependant or otherwise. A non-zero $2 \times 2$ matrix, $A$, is called isotropic or degenerate if $\operatorname{det}(A)=0$ and anisotropic otherwise. So only non-zero anisotropic matrice have inverses.

\section{Lemma 2.2}


A pair of traceless matrices $\mathbf{a}, \mathbf{b}$ is linearly dependant if and only if $\mathbf{a} \times \mathbf{b}=0$.

Proof The proof is clear one way using the antisymmetry of the cross product. Conversely suppose $\mathbf{a} \times \mathbf{b}=0$. Then $(\mathbf{a} \times \mathbf{b}) \times \mathbf{c}=(\mathbf{a} \cdot \mathbf{c}) \mathbf{b}-(\mathbf{b} \cdot \mathbf{c}) \mathbf{a}=0$. This can be made into a linear dependancy by a suitable choice of $\mathbf{c}$, for example if $\mathbf{a} \cdot \mathbf{c} \neq 0$.

As a corollary we have the following

\section{Lemma 2.3}

Two $2 \times 2$ matrices commute if and only their traceless parts are linearly dependant.

Now we look for conditions for the triple of traceless matrices, $\mathbf{a}, \mathbf{b}, \mathbf{a} \times \mathbf{b}$, to be linearly dependant. The required condition is given by the following lemma.

\section{Lemma 2.4}

The traceless matrices $\mathbf{a}, \mathbf{b}, \mathbf{a} \times \mathbf{b}$, are linearly dependant if and only if

$$
\operatorname{det}(\mathbf{a}) \operatorname{det}(\mathbf{b})=(\mathbf{a} \cdot \mathbf{b})^{2} .
$$

This is equivalent to the condition that $\mathbf{a} \times \mathbf{b}$ is isotropic or zero.

Proof Three 3-dimensional vectors are linearly dependant if and only if the determinant they form by rows is zero. In the case of traceless matrices this means the scalar triple product is zero

$$
[\mathbf{a}, \mathbf{b}, \mathbf{c}]=\mathbf{a} \cdot(\mathbf{b} \times \mathbf{c})=0 .
$$

Replacing $\mathbf{c}$ with $\mathbf{a} \times \mathbf{b}$ and expanding out using the triple cross product formula gives the first equation. Using the expansion formulæ

$$
\operatorname{det}(\mathbf{a} \times \mathbf{b})=\operatorname{det}(\mathbf{a}) \operatorname{det}(\mathbf{b})-(\mathbf{a} \cdot \mathbf{b})^{2}
$$

gives the second condition.

We have the following corollary.

\section{Lemma 2.5}

If $\mathbf{a}, \mathbf{b}$ are traceless matrices and $\mathbf{a} \times \mathbf{b}$ has non-zero determinant, then the triple $\mathbf{a}, \mathbf{b}, \mathbf{a} \times$ $\mathbf{b}$, is linearly independant.

3 Solving the Fundamental Equation 
Given a set $X$ let $S$ be an endomorphism of $X^{2}$. Such an $S$ is called a switch if

$1 S$ is invertible and

2 the set theoretic Yang-Baxter equation

$$
(S \times i d)(i d \times S)(S \times i d)=(i d \times S)(S \times i d)(i d \times S)
$$

is satisfied. Switches are used in $[\mathbf{F J K}]$ to define biracks and biquandles by the formula

$$
S(a, b)=\left(b_{a}, a^{b}\right) .
$$

Switches can be used to find representations of the virtual braid groups and invariants of virtual knots and links, see $[\mathbf{F J K}],[\mathbf{B F}],[\mathbf{B u F}]$ and $[\mathbf{F}]$.

We are looking for linear solutions. That is $S=\left(\begin{array}{cc}A & B \\ C & D\end{array}\right)$, where the matrix entries $A, B, C, D$ are elements of $R$, an associative but not necessarily commutative ring and $X$ is a left $R$-module.

The solutions can be divided into two types when the entries are not zero divisors.

\section{The commutative case}

0 :

The identity

1: $\quad S=\left(\begin{array}{cc}0 & B \\ C & 1-B C\end{array}\right)$ or $S=\left(\begin{array}{cc}1-B C & B \\ C & 0\end{array}\right)$

where $B$ and $C$ are arbitary commuting invertible elements.

\section{The non-commutative case}

2 :

$$
S=\left(\begin{array}{ll}
A & B \\
C & D
\end{array}\right)
$$

where $A, A-1, B$ are invertible, $A, B$ do not commute and satisfy the fundamental equation

$$
A^{-1} B^{-1} A B-B^{-1} A B=B A^{-1} B^{-1} A-A
$$

moreover

$$
C=A^{-1} B^{-1} A(1-A), D=1-A^{-1} B^{-1} A B .
$$

There are also similar solutions where $A, D$ and $B, C$ are interchanged. We are only interested in this last case and are therefore looking for $2 \times 2$ matrices $A=a_{0}+\mathbf{a}$ and 
$B=b_{0}+\mathbf{b}$ which satisfy the fundamental equation. Since $A$ and $B$ do not commute $\mathbf{a}$ and $\mathbf{b}$ are linearly independent. As in $[\mathbf{B u F}]$ and $[\mathbf{F}]$ the linear relation

$$
\begin{gathered}
(\operatorname{tr}(A)-\operatorname{det}(A)) \operatorname{det}(\mathbf{b}) \mathbf{a}+(\operatorname{det}(A)-\operatorname{tr}(A))(\mathbf{a} \cdot \mathbf{b}) \mathbf{b} \\
+\left(b_{0}(\operatorname{det}(A)-\operatorname{tr}(A))+2 A \cdot B\right) \mathbf{a} \times \mathbf{b}=0
\end{gathered}
$$

holds.

The paper $[\mathbf{F}]$ has solved the matching solutions. That is solutions where $\operatorname{det}(A)=$ $\operatorname{tr}(A)$ and $A \cdot B=0$. So we are interested in the mismatching solutions. In this case $A, B$ satisfy the fundamental equation and $\mathbf{a}, \mathbf{b}$ and $\mathbf{a} \times \mathbf{b}$ are linearly dependent.

4 Finding Linearly Dependent Triples $\mathbf{a}, \mathbf{b}$ and $\mathbf{a} \times \mathbf{b}$

In this section we find precise conditions for the triple $\mathbf{a}, \mathbf{b}$ and $\mathbf{a} \times \mathbf{b}$ to be linearly dependant. Recall that this happens if $\operatorname{det}(\mathbf{a} \times \mathbf{b})=0$, ie $\mathbf{a} \times \mathbf{b}$ is isotropic. A vector is isotropic if it lies in $X$, the right circular cone $x_{1}^{2}-x_{2}^{2}-x_{3}^{2}=0$.

Let

$$
\mathbf{a} \cdot \mathbb{E} \mathbf{b}=a_{1} b_{1}+a_{2} b_{2}+a_{3} b_{3}
$$

and

$$
\mathbf{a} \times \mathbb{E} \mathbf{b}=\left|\begin{array}{ccc}
i & j & k \\
a_{1} & a_{2} & a_{3} \\
b_{1} & b_{2} & b_{3}
\end{array}\right|
$$

denote "euclidean" scalar and cross product respectively. This is to distinguish them from the "hyperbolic" versions

$$
a \cdot \mathbb{H} \mathbf{b}=a_{1} b_{1}-a_{2} b_{2}-a_{3} b_{3}
$$

and

$$
\mathbf{a} \times_{\mathbb{H}} \mathbf{b}=\left|\begin{array}{ccc}
-i & j & k \\
a_{1} & a_{2} & a_{3} \\
b_{1} & b_{2} & b_{3}
\end{array}\right|
$$

Let $\rho$ be the involution given by $\rho\left(x_{1}, x_{2}, x_{3}\right)=\left(-x_{1}, x_{2}, x_{3}\right)$. Then $X$ is invariant under $\rho$ and $\rho(\mathbf{a} \times \mathbb{H} \mathbf{b})=\mathbf{a} \times_{\mathbb{E}} \mathbf{b}$.

If $\mathbf{c}$ is isotropic let $\mathbf{a}, \mathbf{b}$ lie in the plane $\mathbf{c} \cdot \mathbb{E} \mathbf{x}=0$. This plane meets the cone in the generator containing $\rho(\mathbf{c})$. So $\mathbf{a} \times_{\mathbb{E}} \mathbf{b}$ is parallel to $\mathbf{c}$ and $\mathbf{a} \times \mathbb{H} \mathbb{b}$ is isotropic. This means that the triple $\mathbf{a}, \mathbf{b}, \mathbf{a} \times \mathbb{H} \mathbf{b}$ is linearly dependant. Moreover all examples of such triples are obtained in this way.

From now on $\mathbf{a} \cdot \mathbf{b}$ and $\mathbf{a} \times \mathbf{b}$ will have their original (hyperbolic) meanings. 


\subsection{A Worked Example}

Now we find a generic family of triples containing all the properties needed. This is summed up by the following theorem

Theorem 4.2 Without loss of generality we can assume that $\mathbf{a}$ and $\mathbf{b}$ lie in the plane $x_{1}-x_{2}=0$. The most general examples being

$$
\mathbf{a}=\left(\begin{array}{cc}
a_{3} & 2 a_{1} \\
0 & -a_{3}
\end{array}\right), \quad \mathbf{b}=\left(\begin{array}{cc}
b_{3} & 2 b_{1} \\
0 & -b_{3}
\end{array}\right), \quad \mathbf{a} \times \mathbf{b}=\left(\begin{array}{cc}
0 & 2\left(a_{3} b_{1}-a_{1} b_{3}\right) \\
0 & 0
\end{array}\right) .
$$

$$
\text { So } b_{3} \mathbf{a}-a_{3} \mathbf{b}+\mathbf{a} \times \mathbf{b}=0 \text {. }
$$

Proof Some small lemmas are needed.

\section{Lemma 4.3}

For any traceless matrices $\mathbf{a}, \mathbf{b}, \mathbf{c}$, we have

$$
(\mathbf{a} \times \mathbf{c}) \cdot(\mathbf{b} \times \mathbf{c})=\operatorname{det}(\mathbf{c})(\mathbf{a} \cdot \mathbf{b})-(\mathbf{a} \cdot \mathbf{c})(\mathbf{b} \cdot \mathbf{c})
$$

Proof This is just a routine calculation.

We will use conjugation in the group theoretic sense, (ie. $A$ conjugated by $B$ is $B^{-1} A B$ ). Since the word conjugation is already being used in a rather different sense (analogous to complex conjugation) we will use the term group-conjugation. Note that the set of solutions to the fundamental equation is invariant under group-conjugation.

\section{Lemma 4.4}

The inner product is invariant under group-conjugation. This means that for any $2 \times 2$ matrix $C$ and any tracesless $2 \times 2$ matrices $\mathbf{a}$ and $\mathbf{b}$ we have

$$
C^{-1} \mathbf{a} C \cdot C^{-1} \mathbf{b} C=\mathbf{a} \cdot \mathbf{b}
$$

Proof Tedious but routine calculation using the above lemma.

\section{Lemma 4.5}

Any tracesless $2 \times 2$ matrix is group-conjugate to a matrix of the form $a_{1} \mathbf{i}+a_{2} \mathbf{j}$. In particular any isotropic tracesless $2 \times 2$ matrix is group-conjugate to one of the form $x(\mathbf{i}+\mathbf{j}), x \in F$

Proof If $a_{3}=0$ then we are already there, so we will assume otherwise. 
Case 1: $a_{2}^{2}+a_{3}^{2} \neq 0$.

Consider $C=\left(-a_{2}+\sqrt{a_{2}^{2}+a_{3}^{2}}\right)-a_{3} \mathbf{i}$. Then $C$ is invertible whenever -

$$
\operatorname{det}(C)=2\left(a_{2}^{2}+a_{3}^{2}-a_{2} \sqrt{a_{2}^{2}+a_{3}^{2}}\right)=2\left(\sqrt{a_{2}^{2}+a_{3}^{2}}\right)\left(\sqrt{a_{2}^{2}+a_{3}^{2}}-a_{2}\right) \neq 0
$$

Hence $C$ is invertible. Moreover

$$
C^{-1} \mathbf{a} C=a_{1} \mathbf{i}+\sqrt{a_{2}^{2}+a_{3}^{2}} \mathbf{j}
$$

so we are done.

Case 2: $a_{2}^{2}+a_{3}^{2}=0$ Again $a_{2}$ and $a_{3}$ are not zero and so the underlying field must have a square root of -1 , unique up to multiplication by -1 , which we will call $I$. In fact $I= \pm a_{3} / a_{2}$. Group-conjugating $\mathbf{a}$ by $1+I \mathbf{i}$ gives $a_{1} \mathbf{i}$, as required.

Isotropic vectors are invariant under group conjugation and so by the above we can assume an isotropic vector is of the form $x(\mathbf{i}+\mathbf{j})$. Hence from above $\mathbf{a}$ and $\mathbf{b}$ can be conjugated such that,

$$
a_{1}(\mathbf{i}-\mathbf{j}) \cdot \mathbb{E} \mathbf{a}=a_{1}(\mathbf{i}-\mathbf{j}) \cdot \mathbb{E} \mathbf{b}=0 .
$$

The most general case then is (up to group-conjugation) $\mathbf{a}=a_{1} \mathbf{i}+a_{1} \mathbf{j}+a_{3} \mathbf{k} \mathbf{b}=$ $b_{1} \mathbf{i}+b_{1} \mathbf{j}+b_{3} \mathbf{k}$. Substituting

$$
\mathbf{i}=\left(\begin{array}{cc}
0 & 1 \\
-1 & 0
\end{array}\right), \mathbf{j}=\left(\begin{array}{ll}
0 & 1 \\
1 & 0
\end{array}\right), \mathbf{k}=\left(\begin{array}{cc}
1 & 0 \\
0 & -1
\end{array}\right)
$$

concludes the proof of Theorem 4.2

5 How To Find $A$ and $B$ given Linearly Dependent $\mathbf{a}, \mathbf{b}$ and $\mathbf{a} \times \mathbf{b}$.

In this section we complete our theoretical solution of $\mathbf{a}, \mathbf{b}$ to find $A$ and $B$.

Assume that $\mathbf{a}, \mathbf{b}$ and $\mathbf{a} \times \mathbf{b}$ are linearly dependent. That is for some coefficients not all zero there is a linear relationship

$$
\lambda_{1} \mathbf{a}+\lambda_{2} \mathbf{b}+\lambda_{3} \mathbf{a} \times \mathbf{b}=0
$$

We are assuming that $A$ and $B$ do not commute, so $\mathbf{a}, \mathbf{b}$ are linearly independant and hence $\lambda_{3} \neq 0$ and we can write

$$
\lambda_{1} \mathbf{a}+\lambda_{2} \mathbf{b}+\mathbf{a} \times \mathbf{b}=0
$$

where $\lambda_{1}$ and $\lambda_{2}$ are unique. Both $\lambda_{1}$ and $\lambda_{2}$ cannot be zero for then by our earlier discussion $A$ and $B$ would commute. 
We can obtain information about $\lambda_{1}$ and $\lambda_{2}$ by taking the cross product of $\mathbf{a}$ with $\mathbf{a} \times \mathbf{b}$

$$
\begin{aligned}
\mathbf{a} \times(\mathbf{a} \times \mathbf{b}) & =-\lambda_{1} \mathbf{a} \times \mathbf{a}-\lambda_{2} \mathbf{a} \times \mathbf{b} \\
& =\lambda_{1} \lambda_{2} \mathbf{a}+\lambda_{2}^{2} \mathbf{b} \text { on the one hand, and } \\
& =(\mathbf{b} \cdot \mathbf{a}) \mathbf{a}-(\mathbf{a} \cdot \mathbf{a}) \mathbf{b} \text { by standard expansion rules }
\end{aligned}
$$

Comparing coefficients, we have -

$$
\begin{aligned}
\lambda_{1} \lambda_{2} & =\mathbf{a} \cdot \mathbf{b} \\
\lambda_{2}^{2} & =-\operatorname{det}(\mathbf{a}): \text { and similarly } \\
\lambda_{1}^{2} & =-\operatorname{det}(\mathbf{b})
\end{aligned}
$$

Comparing (2) and (3) with (1) and separating coefficients we get the two equations

$$
\lambda_{i}\left[(\operatorname{det}(A)-\operatorname{tr}(A)) \lambda_{1}+b_{0} \operatorname{det}(A)+2 \lambda_{1} \lambda_{2}\right]=0 \quad i=1,2
$$

Since we cannot have both $\lambda_{1}$ and $\lambda_{2}=0$, else we get a commuting solution, we have -

$$
\begin{aligned}
b_{0} & =\frac{\left(\operatorname{tr}(A)-\operatorname{det}(A)-2 \lambda_{2}\right) \lambda_{1}}{\operatorname{det}(A)} \\
& =\lambda_{1}\left(\frac{2}{a_{0}+\lambda_{2}}-1\right) \\
& =\sqrt{-\operatorname{det}(\mathbf{b})}\left(\frac{2}{a_{0}+\sqrt{-\operatorname{det}(\mathbf{a})}}-1\right)
\end{aligned}
$$

Thus, if $\mathbf{a}$ and $\mathbf{b}$ are such that $\mathbf{a}, \mathbf{b}$ and $\mathbf{a} \times \mathbf{b}$ are linearly dependent, then we can pick any $a_{0}$, not equal to $-\sqrt{-\operatorname{det}(\mathbf{a})}$, then choose $b_{0}$ according to (4), and we will have $A$ and $B$ that satisfy the fundamental equation. Note that the roots will have opposite sign.

To be a switch we also require $B, A, A-I$ and $S$ to be invertible.

Now $A$ is singular if and only if $\operatorname{det}(A)=a_{0}^{2}-\lambda_{2}^{2}=0$ if and only if $a_{0}= \pm \lambda_{2}$ $(A-1)$ is singular if and only if $a_{0}=1 \pm \lambda_{2}$ and $B$ is singular if and only if $b_{0}= \pm \lambda_{2}$ if and only if $\lambda_{1}=0$ or $a_{0}=1-\lambda_{2}$.

\section{Lemma 5.6}


If $A, B$ and $(A-1)$ are invertible then so is $S$

Proof According to $[\mathbf{B F}], S$ is invertible if $\Delta^{\prime}=C^{-1} D-A^{-1} B$ is invertible. Using $C=A^{-1} B^{-1} A(1-A), D=1-A^{-1} B^{-1} A B$ we find that $\Delta^{\prime}=(1-A)^{-1} A^{-1} B(A-1)$.

So we require $\lambda_{1} \neq 0$ and $a_{0} \neq \pm \lambda_{2}, 1 \pm \lambda_{2}$.

5.1 The Worked Example(continued)

We have $\lambda_{1}=b_{3}$ and $\lambda_{2}=-a_{3}$. So $b_{0}=b_{3}\left(\frac{2}{a_{0}-a_{3}}-1\right)$

hence all mismatching solutions are conjugate to ones of the form -

$$
A=\left(\begin{array}{cc}
a_{0}+a_{3} & 2 a_{1} \\
0 & a_{0}-a_{3}
\end{array}\right) \quad B=\left(\begin{array}{cc}
\frac{2 b_{3}}{a_{0}-a_{3}} & 2 b_{1} \\
0 & 2 b_{3}\left(\frac{1}{a_{0}-a_{3}}-2\right)
\end{array}\right)
$$

The matrices $A$ and $A-I$ will be invertible as long as $a_{0} \neq \pm a_{3}, 2+a_{3} 1 \pm a_{3}$.

\section{References}

[As] Helmer Aslaksen, Quaternionic Determinants, Math. Intel. Vol 18 no. 3 (1996)

[F] Roger Fenn, Quaternion Algebras and Invariants of Virtual Knots and Links, part I: the Elliptic case, to appear in JKTR

[BF] A. Bartholomew and Roger Fenn. Quaternionic Invariants of Virtual Knots and Links, to appear in JKTR. Preprint available from

http://www.maths.sussex.ac.uk////Staff/RAF/Maths/Current/Andy/

[BuF] S. Budden and Roger Fenn. The equation

$$
[b,(a-1)(a, b)]=0
$$

and virtual knots and links, Fund Math 184 (2004) pp 19-29.

[C] P. M. Cohn. Algebra vol 3 Wiley 1991.

[FJK] R. Fenn, M. Jordan, L. Kauffman, Biquandles and Virtual Links, Topology and its Applications, 145 (2004) 157-175

[K] L.Kauffman. Virtual Knot Theory, European J. Comb. Vol 20, 663-690, (1999)

[L] T. Y. Lam. The Algebraic Theory of Quadratic Forms, Benjamin (1973) 